\title{
The definition of Harpiola (Vespertilionidae: Murininae) and the description of a new species from Taiwan
}

\author{
HaO-Chi KuO ${ }^{1}$, Yin-Ping FAng ${ }^{2}$, GÁbor Csorba ${ }^{3}$, and Ling-Ling LeE ${ }^{1,4}$ \\ ${ }^{1}$ Graduate Institute of Ecology and Evolutionary Biology, National Taiwan University, \\ 1, Sec 4. Roosevelt Road, Taipei, Taiwan R.O.C. \\ ${ }^{2}$ Department of Biological Resources, National Chiayi University, 300 University Road, Chiayi, \\ Taiwan R.O.C. \\ ${ }^{3}$ Department of Zoology, Hungarian Natural History Museum, H-1083 Budapest, Ludovika tér 2, Hungary \\ ${ }^{4}$ Corresponding author: E-mail: leell@ntu.edu.tw
}

A new species of Harpiola from Taiwan is described based on 11 specimens collected between 1998 and 2004 . Careful examination of these specimens and those of the genus Murina, revealed the valid characters distinguishing Harpiola from Murina, including the enlarged upper incisors, the well developed first premolars in both toothrows with their bulk subequal to canines and the second premolars in the corresponding toothrow, and the strongly bifid lower canine. The new species from Taiwan can be distinguished from Harpiola grisea in India by having different shape of second upper premolar and different structure of first upper molar.

Key words: Harpiola sp. nov., Taiwan, taxonomy

\section{INTRODUCTION}

Within the subfamily Murininae two genera are accepted generally, namely Murina and Harpiocephalus (Corbet and Hill, 1992; Koopman, 1993, 1994; Pavlinov, 2003; Simmons, 2005). The name Harpiola was introduced by Thomas (1915) as a separate genus with Murina grisea Peters, 1872 as its type species. Although Tate (1941) accepted this opinion and provided a brief diagnosis of the genus (characterized by the attachment point of the wing and by dental features), Ellerman and Morrison-Scott (1951) listed this form as a subgenus of Murina, a taxonomic arrangement which was followed subsequently.
The description of the genus Harpiola was based on a single specimen collected from Northwest India and both the wet specimen and its extracted skull are in a very bad condition. As Corbet and Hill (1992: 151) stated "the features upon which Thomas based Harpiola do not appear to be artificial, but to some extent the status of grisea must remain uncertain until further material is obtained." Recently, alongside the description of the second known specimen of $H$. grisea from Mizoram, India, Bhattacharyya (2002) re-evaluated Harpiola and raised once again the taxon to generic rank.

During a series of bat surveys carried out between 1998 and 2004 in mountain areas in Taiwan, 11 Harpiola bats were 
obtained that contributed to the richest collection of specimens of this genus in the world. In this paper, detailed comparisons of these specimens with those of $\mathrm{H}$. grisea and Murina spp. were made to reveal their taxonomic status and to re-define the diagnostic features of the genus Harpiola. Meanwhile, the special zoogeography of Harpiola as well as other mammalian genera was also considered.

\section{Materials AND Methods}

The following comparative material was used: Harpiola grisea: India, $\mathrm{BM}(\mathrm{NH})$ 79.11.21.117 (holotype); Murina aenea: Malaysia, BM(NH) 64.770 (holotype); M. aurata: China, MNHN CG1870-590a (paratype); M. cyclotis cyclotis: India, $\mathrm{BM}(\mathrm{NH})$ 9.4.4.4 (cotype); M. cyclotis peninsularis: Malaysia, $\mathrm{BM}(\mathrm{NH}) 64.771$ (holotype); M. florium: Indonesia, $\mathrm{BM}(\mathrm{NH})$ 63.12.26.14 (holotype); M. hilgendorfi: Japan, HZM 1.2974; M. huttoni: India, BM(NH) 79.11.21.685 (holotype); M. leucogaster rubex: India, $\mathrm{BM}(\mathrm{NH})$ 16.3.25.111 (holotype); M. puta: Taiwan, ZMNTU 1998.7.3; M. rozendaali: Malaysia, $\mathrm{BM}(\mathrm{NH}) 83.360$ (holotype); M. silvatica: Japan, HNHM 2001.38.1; M. suilla: Java, HNHM 2000.13.2; M. tubinaris: Pakistan, HNHM 99.14.6; M. ussuriensis: Russian Federation, ZMMU 96368 (paralectotype).

The museum acronyms mentioned in the text are as follows: $\mathrm{BM}(\mathrm{NH})$ - Natural History Museum, London, formerly British Museum (Natural History); ESRI - Endemic Species Research Institute, Nantou; HNHM - Hungarian Natural History Museum, Budapest; HZM - Harrison Institute, Sevenoaks, formerly Harrison Zoological Museum; MNHN Museum National d'Histoire Naturelle, Paris; NMNS - National Museum of Natural Science, Taichung; ZMNTU - Zoological Museum of National Taiwan University, Taipei; THU — Tunghai University, Taichung; ZMMU - Zoological Museum of Moscow State University, Moscow.

The forearm (FA) measurements were taken from dry or alcohol preserved museum specimens with 0.1 $\mathrm{mm}$ accuracy. The following craniodental measurements were taken to the nearest $0.01 \mathrm{~mm}$ by the authors with digital calipers under a stereomicroscope: total length of skull - from the anterior rim of alveolus of the first upper incisor to the most projecting point of the occipital region; condylobasal length from the exoccipital condyle to the posterior rim of alveolus of the first upper incisor; upper canine width
- taken across the outer borders of upper canines; upper molar width - taken across the outer crowns of the last upper molars; zygomatic width - the greatest width of the skull across the zygomatic arches; mastoid width - the greatest distance accross the mastoid region; postorbital width — the least width of the postorbital constriction; maxillary toothrow length - from the front of upper canine to the back of the crown of the third molar; upper canine-premolar length - the largest distance from the front of the upper canine to the back of the crown of the posterior premolar; length of mandible - from the anterior rim of the alveolus of the first lower incisor to the most posterior part of the condyle; mandibular toothrow length - from the front of the lower canine to the back of the crown of the third lower molar; lower canine-premolar length - the greatest distance from the front of the lower canine to the back of the crown of the posterior premolar; height of the coronoid process - taken perpendicularly from the extremity of the coronoid process to the ramus mandibulae.

\section{Evaluation of The Harpiola Characters}

One of the main diagnostic features attributed to Harpiola is the attachment point of the plagiopatagium. According to Tate (1941), Corbet and Hill (1992), Koopman (1994), and Bhattacharyya (2002), it is attached to the base of the first toe in Harpiola whereas it is attached to the base of the claw of the first digit (the distal phalanx) in Murina. However, when describing M. hilgendorfi, Peters (1880) noted and figured the attachment of the wing as being close to the base of the first toe. This view was supported subsequently by Wallin (1969) and Yoshiyuki (1989). The latter described the Japanese population of $M$. hilgendorfi as having a "plagiopatagium attached to basal portion of first phalanxes of the first toe" (Yoshiyuki, 1989: 216). Ognev (1928) noted a similar point of attachment for $M$. og$n e v i$ and $M$. sibirica (these latter taxa are considered to be synonyms of $M$. hilgendorfi - see Simmons, 2005). However, this character was overlooked by subsequent authors and curiously Tate (1941: 579, 580), although citing Ognev's (1928) statements, 
erroneously listed the attachment point as a differentiating character of Harpiola.

For dental characters, the main distinguishing feature of Harpiola cited by Tate (1941), Corbet and Hill (1992), and Koopman (1994) was the reduction of the metacones of the anterior and middle upper molars. However, these authors were only able to investigate the holotype of $H$. grisea (the single known specimen of the genus at that time) the teeth of which are extremely worn. Bhattacharyya (2002) in his description of a second specimen of this species did not mention the cusp pattern of the molars. Nevertheless, in Harpiola from Taiwan (described herein as new species), the metacones of the first $\left(\mathrm{M}^{1}\right)$ and second $\left(\mathrm{M}^{2}\right)$ molars are well developed and clearly higher than the paracones. Furthermore, Thomas (1915), Corbet and Hill (1992), and Bhattacharyya (2002) all mentioned the highly reduced canines of Harpiola, which are in contrast with the usual specialized canines seen in Murina. However, even higher degree of reduction in the bulk of upper canine can be observed in M. aurata (Maeda, 1980; Corbet and Hill, 1992). Although quantitative comparison and statistical analysis were not applicable because very few specimens of relevant species are available in the museum collections, detailed examination of comparative material in this study revealed that the upper canine of the new species from Taiwan is clearly the highest one in the upper toothrow with the basal area subequal to that of the second premolar, and is not especially reduced as compared to those of M. aurata. As for lower canine, the new species of Harpiola from Taiwan shows higher degree of reduction in both bulk and height than most species of genus Murina except M. aurata. The new species of Harpiola from Taiwan has both basal area and height of lower canine subequal to those of lower second premolar, similar proportions are also found in M. aurata. In conclusion, the degree of reduction of canines in both upper and lower toothrows can not serve as diagnostic characters distinguishing Harpiola from Murina.

The characters of Harpiola (Figs. 1 and 2) which do support its generic distinction are: 1$)$ the upper incisors $\left(\mathrm{I}^{2}\right.$ and $\left.\mathrm{I}^{3}\right)$ are approximately two-third that of the $\mathrm{C}^{1}$ in height; the basal area of second upper incisor $\left(\mathrm{I}^{3}\right)$ is more than two-third that of $\mathrm{C}^{1}$ (in Murina, the height and crown area of $\mathrm{I}^{2}$ and $\mathrm{I}^{3}$ are at most half that of the $\left.\mathrm{C}^{1}\right)$; 2) the basal area of $\mathrm{C}^{1}, \mathrm{P}^{2}$ and $\mathrm{P}^{4}$ are subequal, their height are gradually decreasing; the corresponding teeth are similar in bulk and height in the lower toothrow (in Murina the canine always greatly exceeds the first premolar in height in both toothrows; $\mathrm{P}^{2}$ almost always, clearly smaller than $\mathrm{P}^{4}$ in height); 3) $\mathrm{C}_{1}$ is strongly bifid, and the additional cusp is well developed (in Murina only a small secondary cingular cusp is present). These diagnostic characters can also be seen in the type of $H$. grisea; however, the cusp structure of $\mathrm{C}_{1}$ can hardly be detected due to the very bad condition of the specimen.

\section{Systematic Description}

Harpiola isodon sp. nov.

\section{Holotype}

ZMNTU 1998.5.3, adult ô, dry skin and skull. Collected by T. S. Ding and K. Y. Wang on 2 May 1998.

\section{Type locality}

Hualien County, Jhuosi Township, Yuli Wildlife Refuge, $23^{\circ} 32^{\prime} \mathrm{N}, 121^{\circ} 15^{\prime} \mathrm{E}$, 2,000 m a.s.l.

\section{Paratypes}

ESRI B0358, adult $q$, in alcohol, skull extracted, Taichung County, Tahsuehshan Forest Recreation Area, Tahsuehshan \#210 
logging road, 1,950 m a.s.1.; ESRI B0359, adult + , dry skin and skull, Taichung County, Tahsuehshan Forest Recreation Area, Tahsuehshan \#210 logging road, $1,950 \mathrm{~m}$ a.s.1.; THU B050016, adult $\delta$, dry skin and skull, Chiayi County, Alishan Township, Lulinshan Major Wildlife Habitats, 2,400 m a.s.l.; ZMNTU 2003.8.4, adult $\$$, dry skin and skull, Ilan County, Nanau Township, Nanaunan logging road, $1,000 \mathrm{~m}$ a.s.l.; NMNS 4858, adult + , dry skin and skull, Taichung County, Heping Township, Wuling Farm; NMNS 5741, adult $\hat{\sigma}$, in alcohol, skull extracted, Nantou County, Renai Township, Tsueifong; THU 7385, adult $\widehat{\delta}$, dry skin and skull, Chiayi County, Alishan Township, Lulinshan Major Wildlife Habitats, 2,400 $\mathrm{m}$ a.s.1.; HNHM 2003.36.31, adult $q$, in alcohol, skull extracted, Nantou County, Renai Township, Meifong Farm, $24^{\circ} 06^{\prime} \mathrm{N}, 121^{\circ} 11^{\prime} \mathrm{E}, 2,100 \mathrm{~m}$ a.s.l.; HNHM
2004.19.13. adult ô, skin and skull, Taitung County, Taimali Township, E-ma logging road, $22^{\circ} 37^{\prime} \mathrm{N}, 120^{\circ} 56^{\prime} \mathrm{E}, 1,000 \mathrm{~m}$ a.s.l.; HNHM 2004. 19.15, adult $q$, skin and skull, Chiayi County, Alishan Forest Recreation Area, Sister Ponds, $23^{\circ} 31^{\prime} \mathrm{N}$, $120^{\circ} 48^{\prime} \mathrm{E}, 2,200 \mathrm{~m}$ a.s.1.

\section{Etymology}

The name isodon ('equal-toothed' in English) refers to the subequal basal area of the canines, first and second premolars typical for the genus.

\section{Diagnosis}

This is a medium-sized tube-nosed bat with the forearm length between $31-36 \mathrm{~mm}$ (Table 1). Guard hairs of dorsal fur are with shiny bright golden tips; the uropatagium is densely furred on both sides; wing membrane is attached at the base of first toe.
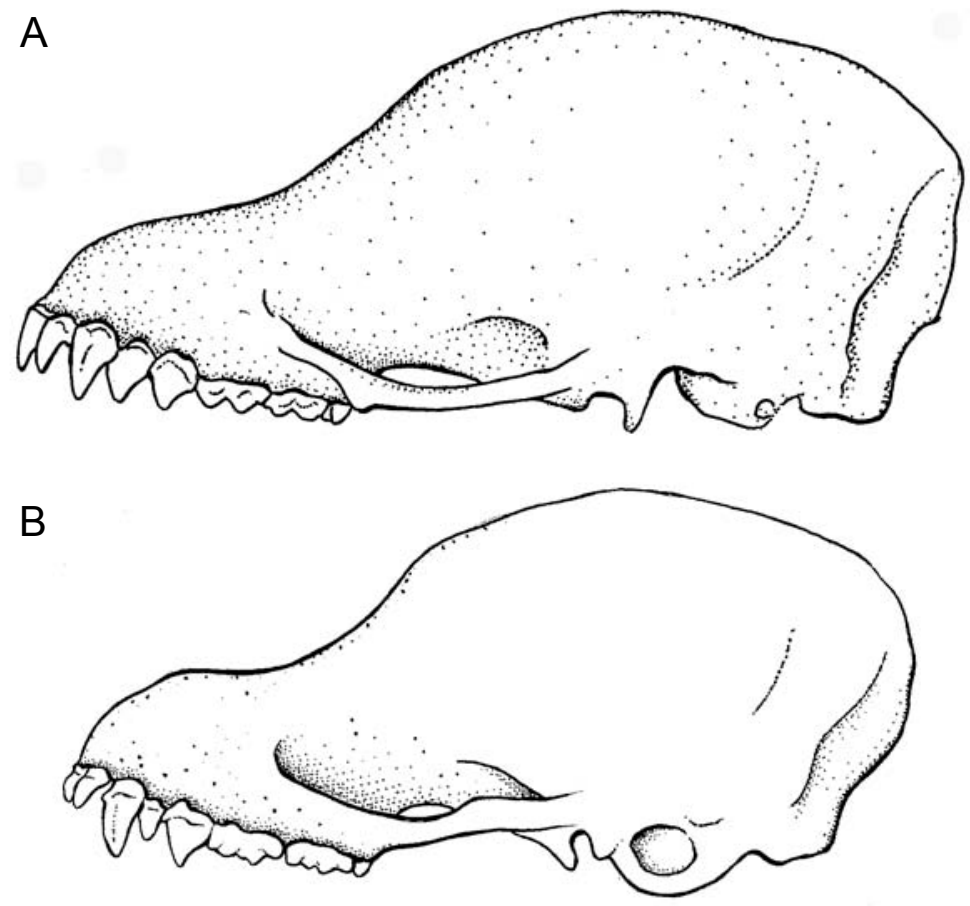

FIG. 1. Lateral views of skulls of: A - Harpiola isodon sp. nov. (paratype HNHM 2003.36.31.) from Taiwan; $\mathrm{B}-$ Murina suilla (type species of the genus, HNHM 2000.13.2.) from Java. Scale $=5 \mathrm{~mm}$ 
The basal area of canines and the premolars are subequal in both toothrows; $\mathrm{P}^{4}$ is wider than long; mesostyles of $\mathrm{M}^{1}$ and $\mathrm{M}^{2}$ are less developed but usually visible, the first upper molar is with a more or less developed postcingular platform.

\section{Description}

The fur on the back is very long (9-10 $\mathrm{mm}$ in length, some guard hair can be as long as $14 \mathrm{~mm}$ ) and woolly; the basal part
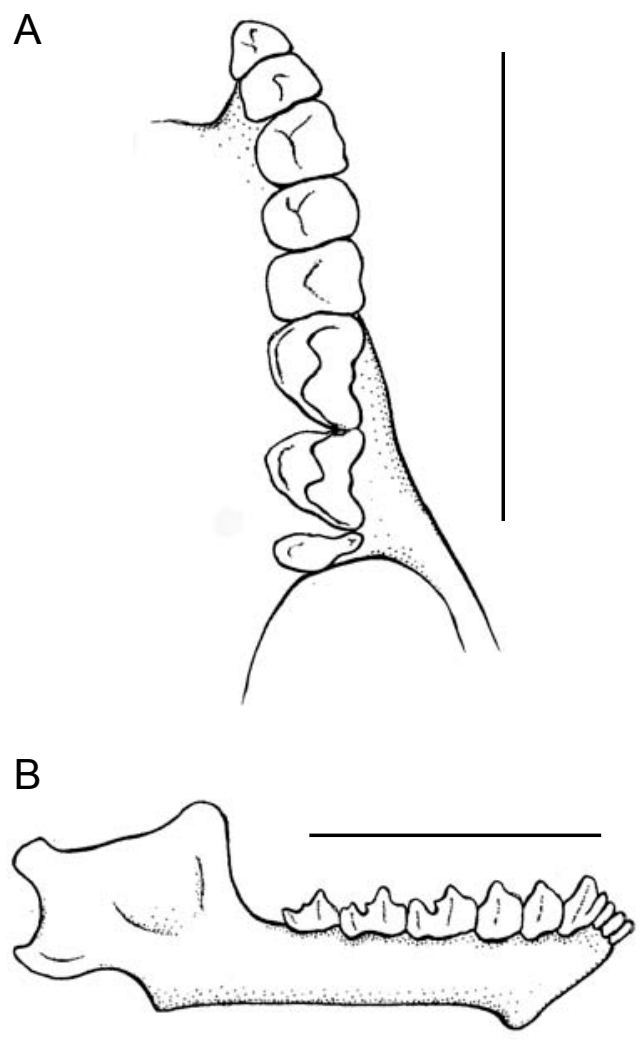

C

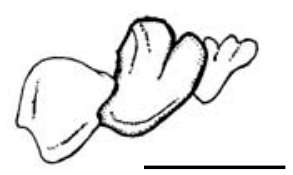

FIG. 2. A - Occlusal view of left upper dentition; $\mathrm{B}$ - lateral view of right mandible; and $\mathrm{C}$ - lingual view of left lower $\mathrm{I}_{3}, \mathrm{C}_{1}$ and $\mathrm{P}_{2}$ teeth of $H$. isodon $\mathrm{sp}$. nov. (paratype, HNHM 2003.36.31.) from Taiwan.

Scales for $\mathrm{A}$ and $\mathrm{B}=5 \mathrm{~mm}$, and for $\mathrm{C}=1 \mathrm{~mm}$ of the individual hair of underfur is dark brown with a bright yellow subterminal band and a dark brown tip. Guard hairs scattered all over the back (including the flanks and the nape) are dark brown in basal four-fifths and with shiny golden yellow tips. The general colouration of back is thus extremely similar to that of $M$. aurata being described as "rich mixture of gold and brown" (Bates and Harrison, 1997: 204). The dorsal side of the tail membrane, the tibia and the foot are all densely and evenly furred including the last caudal vertebra which is free from the uropatagium. The whole length of the forearm, the thumb and even the proximal part of the fifth metacarpal are covered with short golden coloured fur (Fig. 3). In ventral aspect the fur is shorter, dark brown at the base and light brown in the terminal one-third. Some individuals have greyish white hairs predominated along the midline of chest and abdomen. The whole area of the tail membrane is also covered with dense, stiff, silvery grey hairs. The ear is $12.5-13.0 \mathrm{~mm}$ in length, the ear conch is rounded with a very distinct emargination at the upper third of its posterior border; the tragus is medium long $(6.5-8.0 \mathrm{~mm})$ but wide at its base and gradually tapering to the backward-curved tip which just reaches the level of the notch; the base of tragus is with a small tooth-like projection at its outer margin.

The skull is delicately built, the braincase is moderately bulbous, rostral profile is evenly ascending. There is no sagittal crest, the lambdoid crest is medium developed (Fig. 1A). The narial emargination is much longer than wide; there is no basioccipital fissure. Basioccipitale is with well-defined basial pits. The zygomatic arch is weak and slender, almost parallel-sided.

The inner upper incisor $\left(\mathrm{I}^{2}\right)$ is anterior to and only very little longer than the outer upper incisor $\left(\mathrm{I}^{3}\right)$. Both upper incisors are about two-third that of the $\mathrm{C}^{1}$ in height; 
TABLE 1. Selected external and craniodental measurements of $H$. isodon sp. nov. (in mm)

\begin{tabular}{lccc}
\hline \multicolumn{1}{c}{ Parameter } & Holotype & $n$ & Paratypes \\
\cline { 3 - 4 } Forearm length & 31.40 & 9 & $31.00-35.60$ \\
Total length of skull & 15.50 & 10 & $14.76-16.48$ \\
Condylobasal length & 13.87 & 10 & $13.74-14.87$ \\
Upper canine width & 3.65 & 10 & $3.65-4.02$ \\
Upper molar width & 5.17 & 10 & $4.90-5.53$ \\
Zygomatic width & 8.92 & 10 & $8.43-9.35$ \\
Mastoid width & 7.36 & 10 & $7.29-7.96$ \\
Postorbital width & 4.51 & 10 & $4.52-4.84$ \\
Maxillary toothrow length & 4.98 & 10 & $4.97-5.63$ \\
Upper canine-premolar length & 2.34 & 10 & $2.22-2.73$ \\
Length of mandible & 10.28 & 10 & $10.15-11.32$ \\
Mandibular toothrow length & 5.28 & 10 & $5.35-5.90$ \\
Lower canine-premolar length & 2.14 & 10 & $2.02-2.44$ \\
Height of the coronoid process & 3.48 & 10 & $3.36-3.98$ \\
\hline
\end{tabular}

the basal area of second upper incisor is more than two-third that of $\mathrm{C}^{1}$ (Fig. 2A). The basal area of $\mathrm{C}^{1}, \mathrm{P}^{2}$ and $\mathrm{P}^{4}$ are subequal, their height are gradually decreasing. The metacones of $\mathrm{M}^{1}$ and $\mathrm{M}^{2}$ are distinctly higher than the paracones. Second upper premolar is wider than long, $\mathrm{M}^{1}$ and $\mathrm{M}^{2}$ are usually with very small but recognizable mesostyle, the first upper molar is with a definite postcingular platform.

The mandible is slender, the corpus mandibulae is delicate; the symphysis is

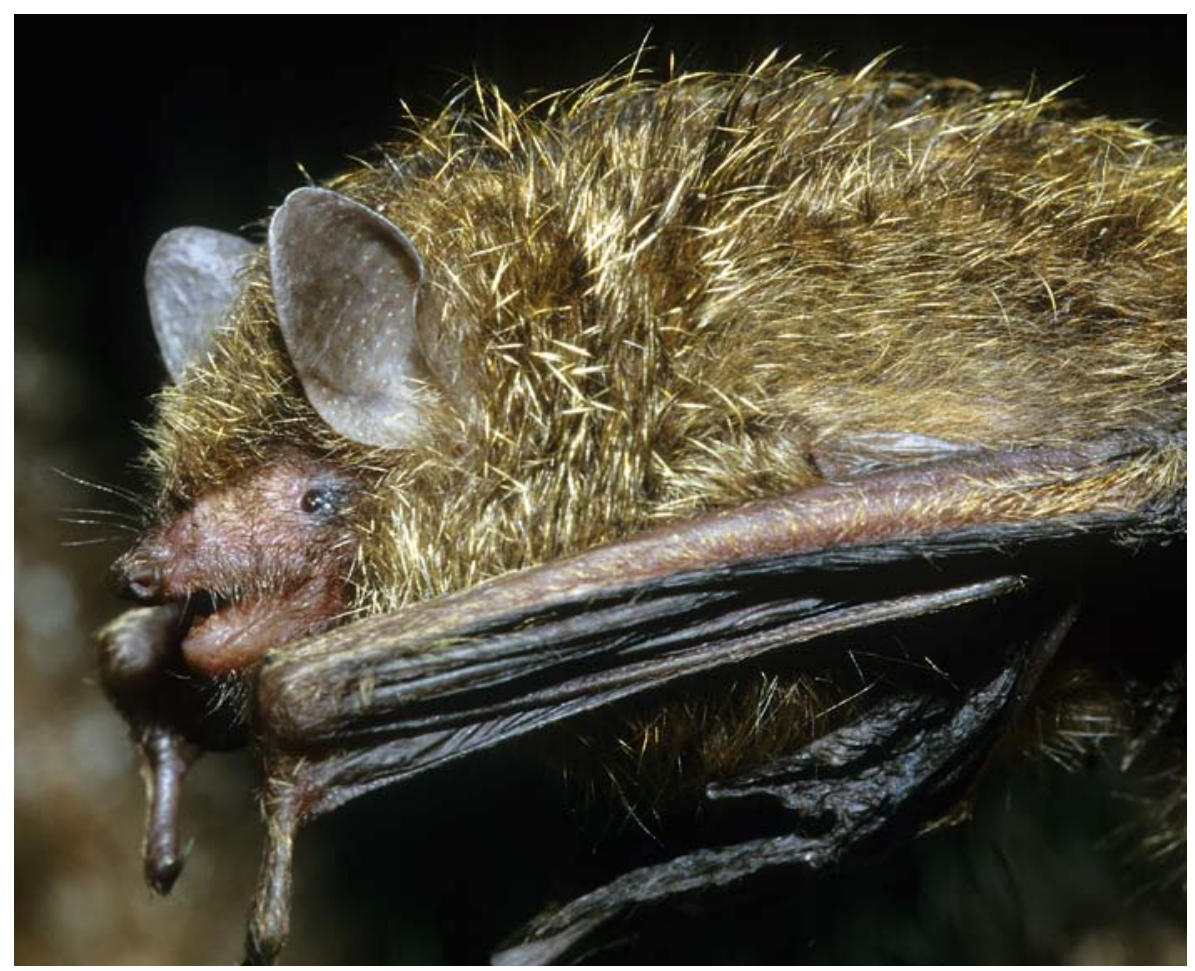

FIG. 3. Photo of a living individual of $H$. isodon sp. nov. (paratype, ESRI B0358) 
long and procumbent, protruding anteriorly; there is a distinct indentation in the corpus in front of the angular process. The coronoid process is low (Fig. 2B). The lower canine has a well developed additional cusp (Fig. 2C); $\mathrm{C}_{1}$ and premolar teeth are similar in both bulk and height among which $\mathrm{C}_{1}$ is slightly less in height and $\mathrm{P}_{2}$ is smaller in basal area. The entoconid of $\mathrm{M}_{1}$ and $\mathrm{M}_{2}$ is lower than the hypoconid but has a distinct cusp and is widely separated from the metaconid; therefore a clear posterior trigonid is present.

\section{Comparisons}

Harpiola grisea, the only other species in the genus with only one specimen before Bhattacharyya (2002), is described by Hutton (1872: 712) as "colour above grey mouse-brown, beneath paler grey." It is the same specimen, however, described by Dobson (1876: 154) as "fur, above, dark brown with yellowish brown extremities; beneath, similar, but the extreme points of the hairs are rather ashy", which is closer to our observation. The external distinction between $H$. grisea and $H$. isodon sp. nov. in Taiwan seems to be the colour of extremities of dorsal hairs, which is dark brown (underfur) or shining golden yellow (guard hairs) in the latter taxon. Interestingly, Bhattacharyya (2002) observed the Mizoram specimen as having hairs on the dorsum with shining golden yellow tips, strikingly similar to those of $H$. isodon. Further materials of $H$. grisea, especially from sites that are close to the type locality, are still needed to make clear the range of variation in pelage colouration of this unique species, or that the Mizoram specimen actually represents a separate taxon.

The condition of the $H$. grisea holotype skull makes any detailed morphological investigation and measuring impossible, and only the shape and size of teeth can be compared. Harpiola grisea differs dentally from $H$. isodon sp. nov. in having a relatively narrow second upper premolar which is almost as long as wide; $\mathrm{M}^{1}$ is with no postcingular platform and $\mathrm{M}^{2}$ is without any trace of mesostyle (Fig. 4) which is usually weak but present in H. isodon.

\section{Natural history}

Individuals were sporadically captured in mountain areas with elevations between 1,000 and 2,400 $\mathrm{m}$ a.s.1. in Taiwan. They occurred mostly in coniferous plantations or mixed forests of coniferous and broadleaf trees with more or less closed canopy. Two of them were found in a tunnel. One female (ESRI B0358), which was caught in May, 2002 had one fetus.

\section{Zoogeography}

Although Taiwan belongs to the Indomalayan zoogeographical region, in the first compilation of the mammal fauna of the island Kuroda (1952: 285) emphasize the fact that "Formosa [...] has rich elements of the Palaearctic or the Himalayan on the mountainous parts". The systematic and taxonomic composition of Taiwanese mammalian fauna has changed a lot in the light of the recent studies, but there are some examples among volant and nonvolant mammal groups which occur only in Taiwan and in the high mountains of
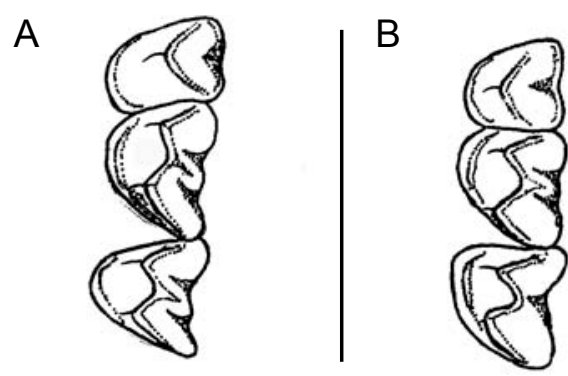

FIG. 4. Occlusal view of the left $\mathrm{P}^{4}, \mathrm{M}^{1}$ and $\mathrm{M}^{2}$ teeth of: $\mathrm{A}-H$. isodon sp. nov. (paratype, HNHM 2003. 36.31.) from Taiwan and $\mathrm{B}-H$. grisea (holotype, $\mathrm{BM}(\mathrm{NH})$ 79.11.21.117) from Uttar Pradesh, India. Scale $=3 \mathrm{~mm}$ 
mainland Asia. This kind of connection is supported by the distribution patterns of shrews (Soriculus and Chodsigoa spp. see Motokawa et al., 1997), mole-shrews (Anourosorex squamipes and A. yamashinai - see Motokawa and Lin, 2002; Motokawa et al., 2004), vesper bats (Arielulus aureocollaris and A. torquatus - see Csorba and Lee, 1999) and voles (Volemys spp.). The species-pair of $H$. isodon and $H$. grisea is an important addition to this list.

\section{ACKNOWLEDGEMENTS}

We are very grateful to Hsi-Chi Cheng (Endemic Species Research Institute, Chichi), Yen-Jean Chen (National Museum of Natural Science, Taichung), Liang-Kong Lin (Tunghai University, Taichung), Paula Jenkins (Natural History Museum, London), Jacques Cuisin (Muséum National d'Histoire Naturelle, Paris) and Paul J. J. Bates (Harrison Institute, Sevenoaks) who kindly provided access to the specimens under their care. György Topál help us with his expert advice and by the preparation of some specimens. The work of HCK, YPF, and LLL was supported by the Nature Conservation Grant of the Council of Agriculture, Taiwan, ROC. The work of GCS was supported by the European Commission's Research Infrastructure Action via the SYNTHESYS Project.

\section{Literature Cited}

BATES, P. J. J., and D. L. HARRISON. 1997. Bats of the Indian subcontinent. Harrisson Zoological Museum Publication, Sevenoaks, 258 pp.

BhatTacharyya, T. P. 2002. Taxonomic status of the genus Harpiola Thomas, 1915 (Mammalia: Chiroptera: Vespertilionidae), with a report of the occurrence of Harpiola grisea (Peters, 1872) in Mizoram, India. Proceedings of the Zoological Society, Calcutta, 55: 73-76.

Corbet, G. B., and J. E. HiLl. 1992. The mammals of the Indomalayan region. Natural History Museum and Oxford University Press, Oxford, 488 pp.

Csorba, G., and L.-L. LeE. 1999. A new species of vespertilionid bats from Taiwan and a revision of the taxonomic status of Arielulus and Thainycteris (Chiroptera: Vespertilionidae). Journal of Zoology (London), 248: 361-367.

Dobson, G. E. 1876. Monograph of the Asiatic Chiroptera, and catalogue of the species of bats in the collection of the Indian Museum, Calcutta. Trustees of the Indian Museum, London, $228 \mathrm{pp}$.

ElLERMAN, J. R., and T. C. S. Morrison-SCOTT. 1951. Checklist of Palaearctic and Indian mammals 1758 to 1946. Trustees of the British Museum (Natural History), London, $810 \mathrm{pp}$.

HutTon, T. 1872. On the bats of the north-western Himalayas with notes and corrections in nomenclature by W. Peters, C.M.Z.S. Proceedings of the Zoological Society of London, 1872: 690-714.

Koopman, K. F. 1993. Order Chiroptera. Pp. 137-241, in Mammal species of the word: a taxonomic and geographic reference. 2nd edition (D. E. WILSON and D. M. REEDER, eds.). Smithsonian Institution Press, Washington D.C., 1206 pp.

Koopman, K. F. 1994. Chiroptera: systematics. Handbook of Zoology. Mammalia, part 60. Walter de Gruyter, Berlin, 217 pp.

KuRODA, N. 1952. Mammalogical history of Formosa, with zoogeography and bibliography. Quarterly Journal of Taiwan Museum, 5: 267-304.

MaedA, K. 1980. Review on the classification of little tube-nosed bats, Murina aurata, group. Mammalia, 44: 531-551.

MotoKawa, M., and L.-K. Lin. 2002. Geographic variation in the mole-shrew Anourosorex squamipes. Mammal Study, 27: 113-120.

Motokawa, M., H.-T. Yu, Y.-P. Fang, H.-C. Cheng, L.-K. Lin, and M. HARADA. 1997. Re-evaluation of the status of Chodsigoa sodalis Thomas, 1913 (Mammalia: Insectivora: Soricidae). Zoological Studies, 36: 42-47.

Motokawa, M., M. Harada, L.-K. Lin and Y. WU. 2004. Geographic differences in karyotypes of the mole-shrew Anourosorex squamipes (Insectivora, Soricidae). Mammalian Biology, 69: 197-201.

OGNEv, S. I. 1928. Mammals of Eastern Europe and Northern Asia. Volume 1. Insectivora and Chiroptera. Glavnauka, Moscow, 631 pp. [in Russian].

Pavlinov, I. Y. 2003. Systematics of recent mammals. Moscow University Publisher, Moscow, 296 pp. [in Russian].

Peters, W. 1880. Mittheilung über die von Hrn. Dr. F. Hilgendorf in Japan gesammelten Chiropteren. Monatsberichte der Königlich Preussischen Akademie der Wissenschaften, 1880: 23-25.

Simmons, N. B. 2005. Order Chiroptera. Pp. 312-529, in Mammal species of the Word: a taxonomic and geographic reference. 3rd edition (D. E. Wilson and D. M. ReEDER, eds.). The Johns Hopkins University Press, Baltimore, $2142 \mathrm{pp}$. 
Tate, G. H. H. 1941. Results of the Archbold expeditions no. 40. Notes on Vespertilionid bats of the subfamilies Miniopterinae, Murininae, Kerivoulinae and Nyctophilinae. Bulletin of the American Museum of Natural History, 78: 567-597.

THOMAS, O. 1915. A special genus for the Himalayan bat known as Murina grisea. Annals and Magazines of Natural History, 8, 16: 309-310.

WALLIN, L. 1969. The Japanese bat fauna. Zoologiska Bidrag från Uppsala, 37: 223-440.

YoshIYUKI, M. 1989. A systematic study of the Japanese Chiroptera. National Science Museum, Tokyo, $242 \mathrm{pp}$.

Received 02 September 2005, accepted 10 December 2005 